\title{
Effect of baffles geometry of the flocculation basin on the turbulence behavior using Comsol multiphysics technique
}

\author{
Ali Salim Joodi \\ Al_ Mustansiriya University, Collage of Engineering, Environmental Eng.
}

Rec. 6 Aug, 2012 Accpt. 1 Oct, 2012

\begin{abstract}
The purpose of flocculation basin is to accelerate the pace at which the particles collide, causing the agglomeration of electrolytically destabilized particles into settable and filterable sizes. One of the most important factors that influence the particles collision in a baffled hydraulic flocculator is the geometry of baffles. In this work, two dimensional mathematical model was established by COMSOL Multiphysics technique to investigate the influence of baffles geometry on the behavior of turbulence in hydraulic flocculator and consequently on the rate of collision of particles. K- $\varepsilon$ turbulent model will be used to determine the variation of the velocity filed and turbulent kinetic energy $(\mathrm{k})$ along the baffled flocculation basin. The increasing in the baffle length causes an increase in the turbulence structure along the basin. Decrease of the turbulence kinetic energy was observed with increase in the baffle thickness. The model was very sensitive to the baffle tip shape, when the tip shape is semi circle more turbulence is produced along the basin as that when the tip shape is rectangular. The model is sensitive to the baffle number, but it is not sensitive to the location of water inlet. Any variation on the value of inlet water velocity has a great influence on the turbulence structure of the water transporting the particles.
\end{abstract}

Key words: Hydraulic flocculator, turbulence kinetic energy, baffles geometry, COMSOL multiphysics.

\section{Introduction}

In a water treatment plant, the purpose of flocculation basin is to accelerate the pace at which the particles collide, causing the agglomeration of electrolytically destabilized particles into settable and filterable sizes. The hydraulic flocculator has many advantages such as low operating cost, simple operation and maintenance, common use and well-established design methodology. Typical arrangements for hydraulic flocculators are paddle wheels on horizontal shafts, while at least three consecutive compartments are required to minimize short circuiting. Though the compartments are separated by perforated baffle, the flow of the flocculation basin is a non-uniform flow (Cho, Y. et al., 2010). The efficiency of the basin is affected by the inlet energy, turbidity, water temperature and weather conditions. The inlet kinetic energy is the most important factor among the influence items because it is an indicator to the turbulent in the basin and consequently an indicator to the collision rate between particles (McConnachie, G.L. 1993;
Haarhoff, J. 1998; Haarhoff, J and van der Walt, J. 2001). However, the inlet kinetic energy is affected by the shape and composition of baffle wall in the hydraulic flocculation basin (McConnachie, G.L. 1991). The study on the geometry of the hydraulic flocculation basin and flow conditions in the basin is very important to facilitate an inducement of uniformity in the flocculation basin and to achieve an improvement in the efficiency of the basin. Hydraulic flocculation geometry includes the number and spacing of baffles, the length of the gap at the baffle ends, and the degree to which adjoining baffles overlap. In an earlier, paper (Arboleda-Valencia, J. 1986; Haarhoff, J. 1998). these variables were systematically reduced to a number of critical ratios, and a comprehensive mathematical framework was presented whereby hydraulic flocculators can be designed once these ratios are fixed. (Haarhoff. J and van der Walt, J. 2001). investigated these variables by computational fluid dynamics (CFD) software. They found that these variables affect on the turbulent 
kinetic energy and consequently on the velocity gradient in the basin.

The first objective of the present work is to demonstrate that COMSOL Multiphysics technique is capable to simulate the flow behavior in a hydraulic flocculator realistically. In this sens, two dimensional mathematical model will be established. K- $\varepsilon$ turbulent model will be used to determine the variation the velocity filed, turbulent kinetic energy $(\mathrm{k})$, and dissipation rate of turbulent kinetic energy $(\varepsilon)$ along the baffled flocculation basin. The second aim of this paper is to exploit the mathematical model to predict the effect of the geometry of the hydraulic flocculation basin and flow conditions on the variation of turbulent kinetic energy and consequently on the rate of collision between particles.

\section{Mathematical formulation:}

To describe water flow behavior in a hydraulic flocculation basin, coupling between continuity equation and K- $\varepsilon$ turbulent model is utilized (Bhargava. D.S. and Ojha. C.S.P. 1993; McConnachie. G.L. et al., 1999; McConnachie. G.L., Liu, J., 2000). These equations can be written as:

$$
\begin{aligned}
& \rho \frac{\partial U}{\partial t}-\nabla \cdot\left[\left(\mu+\rho \frac{c_{\mu}}{\sigma_{k}} \frac{k^{2}}{\varepsilon}\right) \nabla U\right]+\rho U \cdot \nabla U+\nabla P= \\
& \nabla U=0
\end{aligned}
$$

Where $\rho$ denotes the water density $\left(\mathrm{kg} / \mathrm{m}^{3}\right)$, $\mathrm{U}$ represents the average water velocity $(\mathrm{m} / \mathrm{s}), \mu$ is the dynamic viscosity $(\mathrm{kg} /(\mathrm{m} \cdot \mathrm{s}))$, $\mathrm{P}$ is the pressure $(\mathrm{Pa}), \mathrm{k}$ refers to the turbulence kinetic energy $\left(\mathrm{m}^{2} / \mathrm{s}^{2}\right), \varepsilon$ is the dissipation rate of turbulent kinetic energy $\left(\mathrm{m}^{2} / \mathrm{s}^{3}\right)$, and $C_{\mu}$ and $\sigma_{k}$ are model constants. The turbulence kinetic energy is found by solving:

$\rho \frac{\partial k}{\partial t}-\nabla \cdot\left[\left(\mu+\rho \frac{C_{\mu}}{\sigma_{k}} \frac{k^{2}}{\varepsilon}\right) \nabla k\right]+\rho U . \nabla k=0.5 \rho C_{\mu} \frac{k^{2}}{\varepsilon}(\nabla U)^{2}-$

$\rho \varepsilon$

In addition the dissipation rate of turbulent kinetic energy by solving:

$\rho \frac{\partial \varepsilon}{\partial t}-\nabla \cdot\left[\left(\mu+\rho \frac{C_{\mu}}{\sigma_{\varepsilon}} \frac{k^{2}}{\varepsilon}\right) \nabla \varepsilon\right]+\rho U . \nabla \varepsilon=0.5 \rho C_{\varepsilon 1} C_{\mu} k(\nabla U)^{2}-$

$\rho C_{\varepsilon 2} \frac{\varepsilon^{2}}{k}$

Other researchers calculated the constants of the above equations in practically as shown in table (1).

\begin{tabular}{|c|c|c|c|c|c|}
\hline Constant & $\boldsymbol{C}_{\boldsymbol{\mu}}$ & $\boldsymbol{C}_{\boldsymbol{\varepsilon} \mathbf{1}}$ & $\boldsymbol{C}_{\boldsymbol{\varepsilon} \mathbf{2}}$ & $\boldsymbol{\sigma}_{\boldsymbol{k}}$ & $\boldsymbol{\sigma}_{\boldsymbol{\varepsilon}}$ \\
\hline Value & 0.09 & 1.44 & 1.92 & 1.0 & 1.3 \\
\hline
\end{tabular}

Table (1): Model constants (Haarhoff. J and van der Walt. J, 2001; Cho. Y. et al., 2010).
Geometry of flocculation basin and boundary conditions:

The hydraulic flocculation basin is a rectangular in the present work with six baffles, as shown in fig (1). Three baffles for each side of the wall. Six variables are required to define in hydraulic flocculator.
$\mathrm{L}^{\prime}$ : Basin length
W: Basin width
L: Length of the baffle.
T: Baffle thickness.

S: Slot width (distance between the baffle tip and the wall).

$\mathrm{N}$ : Baffle number.

The flocculator layout geometry is shown in figure (1).

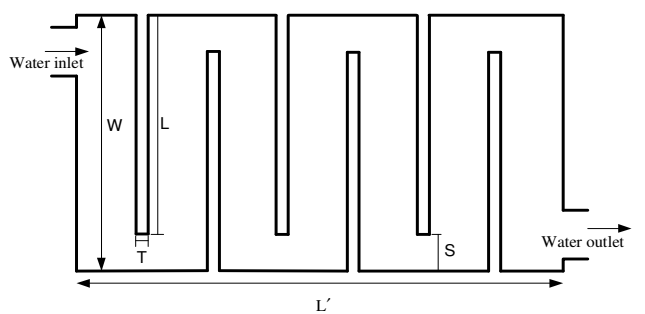

Fig (1): The geometry of baffled flocculation basin

In the present work, $\mathrm{K}-\varepsilon$ turbulent model was used to determine the variation in local the velocity filed, turbulent kinetic energy (k) and dissipation rate of turbulent kinetic energy ( $(\varepsilon)$. K- $\varepsilon$ turbulent model has been applied for computational fluid dynamic (CFD) simulation of water treatment flow (Bhargava. D.S and Ojha. C.S.P. 1993; McConnachie. G.L et al., 1999; McConnachie. G.L., Liu, J., 2000). An immediate benefit of the realizable K- $\varepsilon$ turbulent model is that it more accurately predicts the spreading rate of both planar and round jets. It is also likely to provide superior performance for flows involving rotation, boundary layers under strong adverse pressure gradients, separation, and recirculation (Van der Walt, 1998; Otto, H. 1998; Cho, Y. 2010). The assumptions used for solving the equations included: (1) steady state operation; (2) turbulent flow regime (Realizable K- $\varepsilon$ turbulent model) and (3) Non-slip boundary conditions at wall surfaces and baffles (Cho, Y. 2010). The boundary conditions of the inlet and outlet of the basin were selected as the value of the velocity in the inlet location and zero pressure at the outlet location. The model area is divided into the triangles 
plane mesh. The number of mesh is increased at the tip of baffle to increase the accuracy around baffles, as shown in fig (2). The total number of elements and nodes are 3506 and 1978, respectively. The mathematical model is solved using the commercial COMSOL Multiphysics software.

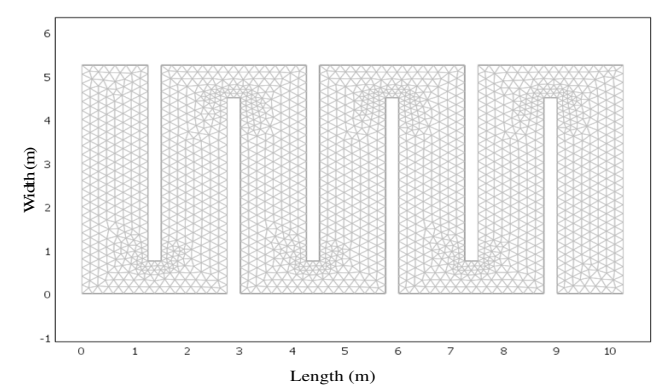

Fig (2): Triangles plane mesh layout in hydraulic flocculator.

Comparison between the results of CFD modeling and COMSOL Multiphysics:

One of the objectives of this research is to demonstrate that COMSOL Multiphysics technique is capable to simulate the flow behavior in a hydraulic flocculator realistically. In this sens, a comparison between the results obtained by Haarhoff, J. and van der Walt, J. 2001 and the results of COMSOL Multiphysics is taken into account. Their model is based on computational fluid dynamic (CFD) software. To provide this comparison, same parameters values were used in this work. Figure (3) shows a comparison between simulated results by CFD software and COMSOL Multiphysics.

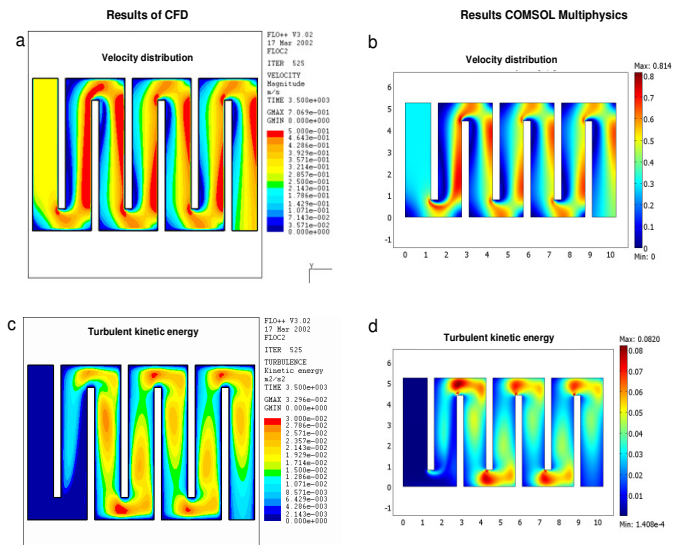

Fig (3): Comparison between the velocity distribution and turbulent kinetic energy from CFD and COMSOL Multiphysics softwares.

From fig ( $3 a$ and $3 b$ ), it can be found that the maximum velocities simulated by CFD analysis and COMSOL Multiphysics are 0.5 and $0.8 \mathrm{~m} / \mathrm{s}$, respectively. From fig ( $3 \mathrm{c}$ and $3 d$ ), it can be found that the maximum turbulent kinetic energy simulated by CFD analysis and COMSOL Multiphysics are 0.03 and $0.08 \mathrm{~m}^{2} / \mathrm{s}^{2}$, respectively. Note that although the result in fig (3) reflects different color, the color scales are similar. These results indicate that COMSOL Multiphysics provides a realistic approximation of the actual flow pattern.

\section{Results and discussion:}

In this research, COMSOL Multiphysics was shown to have powerful potential for the analysis of hydraulic flocculators. Data shown in Table (2) is used to determine the behavior of velocity distribution, turbulent kinetic energy $(\mathrm{k})$ and dissipation rate of turbulent kinetic energy $(\varepsilon)$.

\begin{tabular}{|l|l|l|l|l|l|}
\hline parameter & Basin length & Basin width & L & T & N \\
\hline value & $10.2 \mathrm{~m}$ & $5.2 \mathrm{~m}$ & $4.45 \mathrm{~m}$ & $0.25 \mathrm{~m}$ & 6 \\
\hline
\end{tabular}

Table (2). Details of hydraulic flocculator.

Figure (4a) reveals that high velocities are experienced after each baffle end. This can be attributed to two reasons. The first is the small distance between baffle end and the wall of basin, and the second is the change of direction velocities. The recirculation zone after each baffle extends a considerable distance beyond the end of the baffle, as shown in figure (4b).

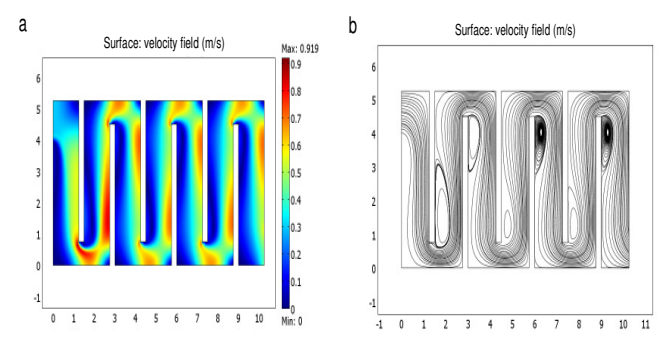

Fig (4): Surface velocity distribution (left) and velocity stream lines (right). 
The baffles geometry is primarily responsible for adding large-scale turbulence to the flocculator (Haarhoff, $\mathrm{J}$ and van der Walt, J. 2001). Figure (5a) shows the spatial variation of turbulent kinetic energy that relates to the production of turbulence. It is clearly observed that turbulence is generated when water is forced between the tip of the baffle and the basin wall. Also, the turbulence begins decreasing as the water moves downstream along the straight channel. Figure (5b) shows the dissipation rate of turbulent kinetic energy that relates to the length scale of eddies that are formed in the turbulent flow field. It is evident that large eddies can be expected to form at the tip of the baffle. Large eddies will then cascade and shed smaller eddies as the water moves downstream along the straight channel.

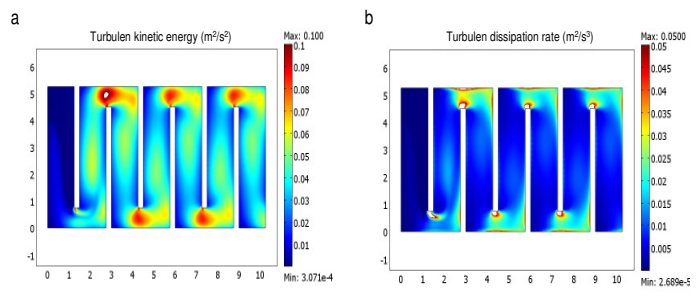

Fig (5): Variation of turbulent kinetic energy and dissipation rate.

\section{Sensitivity analysis}

A sensitivity analysis was the process of varying model parameters over a reasonable range and observing the relative change in model response. Typically, the observed change in turbulent kinetic energy was noted. Then, the objective is to provide more turbulence to increase the potential of collision between particles. For good comparison between figures, we will fix the scale of turbulent kinetic energy between 0.000001 and $0.1 \mathrm{~m}^{2} / \mathrm{s}^{2}$.

\section{Baffle length ( $L)$}

Figure (6) presents the behavior of turbulent kinetic energy with different length of the baffle. In this context, we will use the baffle length ratio $(\mathrm{L} / \mathrm{W})$, which is define that the ratio of the baffle length to the basin width. Three values of ratio were selected $0.66,0.76$ and 0.86 . Generally, it can be observed that the amount of turbulence at the end of baffle is reduced with decrease the baffle length. This can be attributed to the distance between the trip of baffle and the basin wall which is increased with decrease of baffle length and consequently decrease in the velocities and turbulence. At the downstream along the channel, the amount of turbulence disappears gradually with decreasing in the baffle length. The collision of particles is increased with turbulence increase and vice versa. Then in this model, the best results are provided when L/W = 0.86

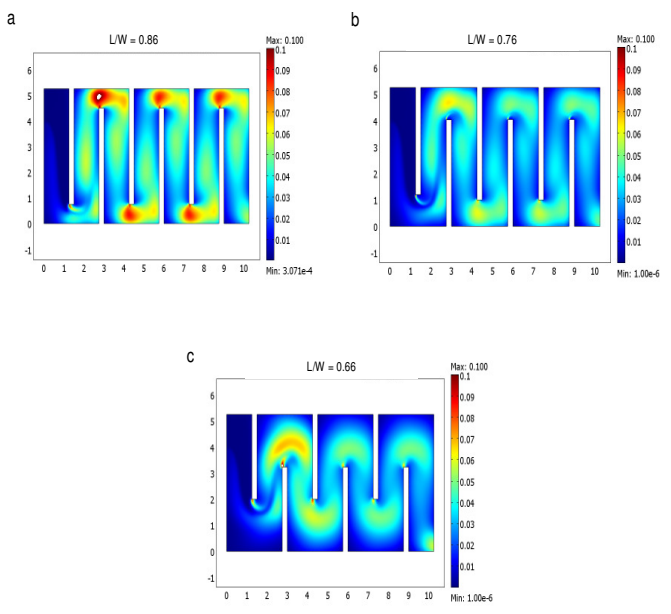

Fig (6): Variation of turbulent kinetic energy as a function of baffle length.

Thickness of baffle (T):

Effect of the baffle thickness is illustrated in fig (7). The baffle thickness ratio $\left(\mathrm{T} / \mathrm{L}^{\prime}\right)$ will be applied. It is define that the ratio of the baffle thickness to the basin length. Three values were selected in this work $0.025,0.05$ and 0.075 . Reducing the baffle thickness causes an increase in the turbulence around the end of baffle as a result to the water velocities increase. Moreover, when the baffle thickness increases, the turbulence eddies begin decreasing as the water moves downstream along the straight channel and vice versa.

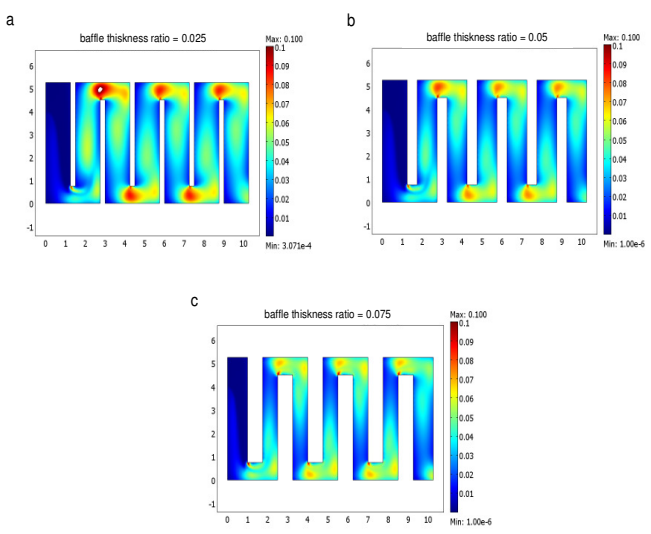

Fig (7): Variation of turbulent kinetic energy as a function of baffle thickness. 


\section{Shape of baffle tip}

In this paper, the shape of baffle tip was investigated to show his effect on the turbulence behavior along the hydraulic flocculator. In this context, two tips were investigated. The first is rectangular and the second is semi circle, as shown in fig (8). Tip shape parameter shows the major effect on the variation of turbulent kinetic energy. When the tip baffle is rectangular (fig 8a), the water is not much forces as it is in the case when the tip is semi circle (fig 8b), and by consequence more turbulence around the end of baffle in the case of semi circle tip as that when the tip is rectangular. This is as a result to the water velocity behavior around the end of baffle. The velocity increases in the case of semi circle tip as that in the case of rectangular tip. Furthermore, the amount of turbulence is not disappearing at the downstream along the channel in the case of semi circle tip. Then, from these results, it can be said that the case of semi circle tip is better than the other case to generate more turbulence along the basin.

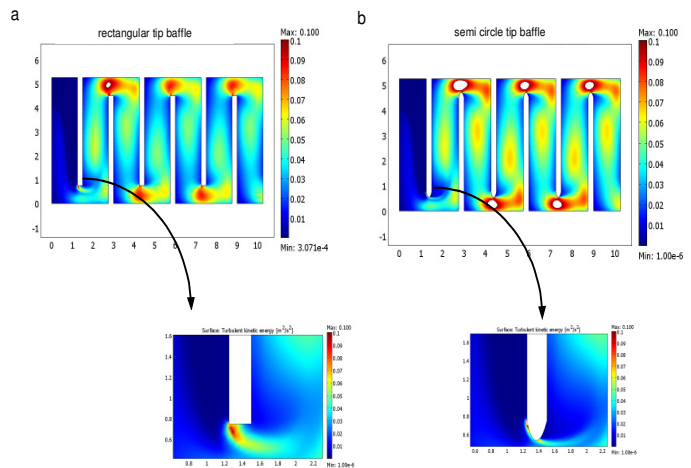

Fig (8): Variation of turbulent kinetic energy as a function of tip baffle shape.

\section{Baffles number:}

The effect of baffles number on the turbulent kinetic energy is presented in fig (9). The increase in the baffles number means a decrease in the channel width. This decrease in the channel width prevents the large eddies of turbulence to cascade more downstream along the channel. The comparison between fig (9a) and (9b) shows that larger volumes of turbulent kinetic energy are formed in the downstream of channels with baffled number increase until the limit in which the width of channel does not allow to continue the eddies along the channel . This can be attributed to the basin length. Then in this model when the length is
$10.2 \mathrm{~m}$, the best results are created when the number of baffles is six compared with another cases. The optimum number of baffles is determined according to the length of the basin. Globally, the effect of baffle number on the behavior of turbulence in the hydraulic flocculator is very considerable.
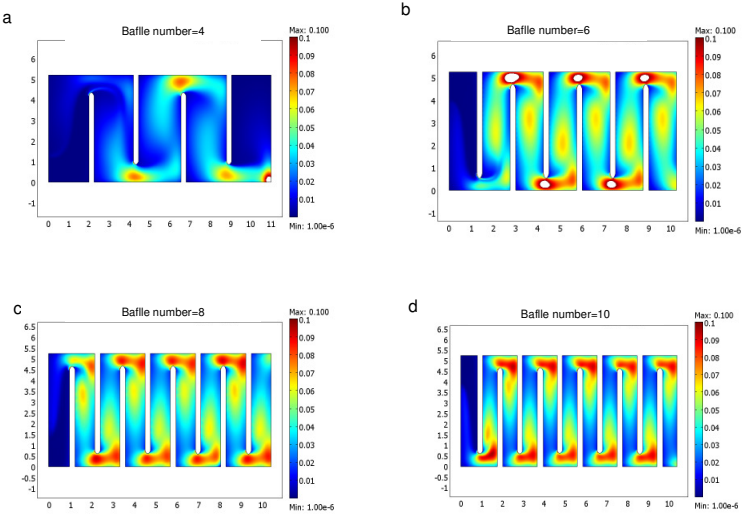

Fig (9): Variation of turbulent kinetic energy as a function of baffle number.

\section{Water flow velocity and inlet location:}

Figure (10) presents the effect of the variation of water velocity inlet on the distribution of turbulent kinetic energy. By the comparison between fig (10a, b and c), it can be observed that increasing of water velocity inlet leads to an increase in the large scale turbulence along the basin.

Figure (11) shows the effect of inlet location on the distribution of turbulent kinetic energy. It can be seen that, the effect of inlet location is not significant on the behavior of water flow along the basin. In spite of there are more turbulence eddies in the first channel in the case when the inlet is perpendicular to the baffle compared with that when the inlet is parallel to the baffle.

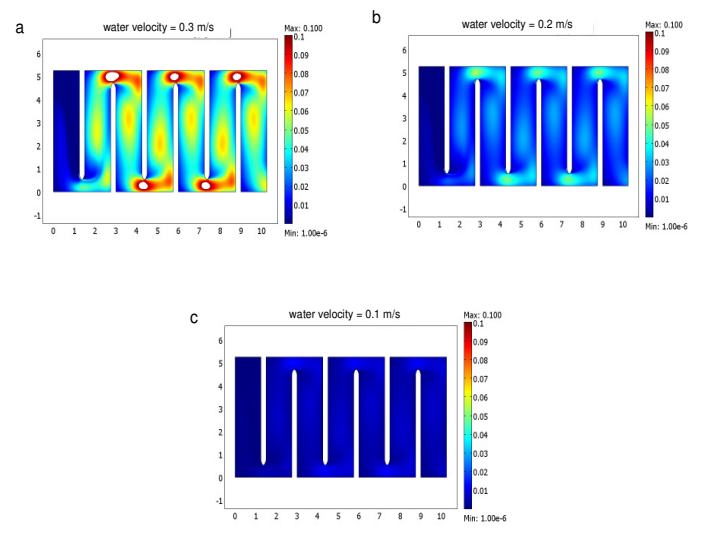

Fig (10): Variation of turbulent kinetic energy as a function of inlet water velocity. 

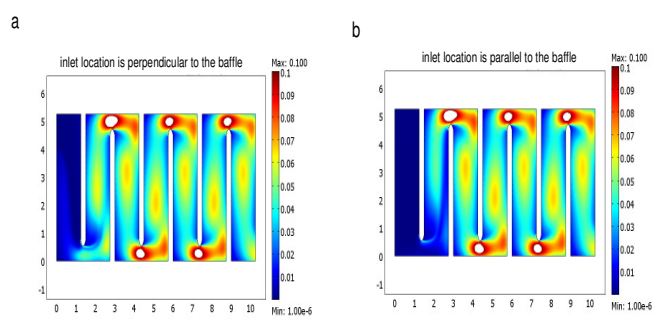

Fig (11): Variation of turbulent kinetic energy as a function of inlet location.

\section{Conclusion:}

Two-dimensional mathematical model is established in the present paper to simulate the water flow behavior in a baffle type of hydraulic flocculator. K- $\varepsilon$ turbulent model was used to determine the variation in local the velocity filed and turbulent kinetic energy (k). K- $\varepsilon$ turbulent model has been applied for computational fluid dynamic (CFD) simulation of water treatment flow. By the comparison between our results and previous results, we concluded that the COMSOL Multiphysics finite element analysis software was capable to simulate the behavior of turbulence along the hydraulic flocculator. Sensitivity analysis was taken into account in order to investigate the effect of geometry and hydraulic parameters on the turbulence behavior within the flocculator basin. The comparison between figures was depended on the turbulent kinetic energy because it is considered as a measure of the turbulence (Haarhoff. J and van der Walt. J, 2001).

The sensitivity analysis has shown that the model is very sensitive to the variation of baffle geometry. The increasing in the baffle length causes an increase in the turbulence structure along the basin. Any increase in the length of the baffle causes increase in the distribution of turbulence along the basin. A decrease in the turbulence kinetic energy was observed with increase in the baffle thickness. The model indicates that the best ratio between the baffle thickness and the basin length is 0.025 . The model is very sensitive to the shape of the baffle tip, because when the tip shape is semi circle more turbulence is produced along the basin as that when the tip shape is rectangular. Then, it can be recommended to construct the tip shape with semi circle. The model is sensitive to the baffle number. However, the baffle number is a function to the basin length, in this model the best results are provided when the baffle number is 6 . Also, the model is not sensitive to the location of inlet water velocity because the results are nearly the same when the inlet location is perpendicular or parallel to the baffle. Any variation on the inlet water velocity value has a great influence on the turbulence structure of the water transporting the particles. Finally, we don't have any solid data about the limit values for the maximum value of turbulence in the baffled hydraulic flocculator.

\section{References:}

Arboleda Valencia, J. (1986). A new approach to treatment plant design and construction. J. American Water Wks. Assoc. 78 (7), 92-105.

Bhargava, D.S. and Ojha, C.S.P. (1993). Models for the design of flocculating baffled channels. J. Water Res. 27 (3), 465-475.

Cho, Y., Yoo, S., Yoo, P. and Kim, C. (2010). Evaluation of the effect of baffle shape in flocculation basin on hydrodynamic behavior using computational fluid dynamics. Korean, J. Chemical. Eng, 27 (3), 874-880.

Haarhoff, J. (1998). Design of around-theend hydraulic flocculators. Aqua 47 (3), 142-152.

Haarhoff, J. and Van der Walt, J. (2001). Towards optimal design parameters for around-the-end hydraulic flocculators. Journal of Water Supply: Research and Technology. 50 (3), 149-159.

McConnachie, G.L. (1991). Turbulence intensity of mixing in relation to flocculation. J. Environmental. Eng. 117(6), 731-750.

McConnachie, G.L. (1993). Water treatment for development countries using baffled-channel hydraulic flocculation. Proc. Inst. Civ. Eng. Water, Maritime \& Energy 101, 5561.

McConnachie, G.L., Folkard, G.K., Mtawali, M.A. and Sutherland, J.P. (1999). Field trials of appropriate hydraulic flocculation processes. Water Res. 33(6), 1425-1434. 
McConnachie, G.L. and Liu, J. (2000). Design of baffled hydraulic channels for turbulence-induced flocculation. Water Res. 34 (6), 1886-1896.

Otto, H. (1998). Flow Patterns in Baffled Channels. Project Investigation for B.Eng degree, Rand Afrikaans University, South Africa.

Rebhun, M. and Argaman, Y. (1965). Evaluation of hydraulic efficiency of sedimentation basins. San. Eng. ASCE 91 (5), 37-45.

Van der Walt, J. (1998). The application of computational fluid dynamics in the calculation of local $G$ values in hydraulic flocculators. Proceedings of the Biennial Conference of the Water Institute of Southern Africa, 1998 Cape Town, South Africa. Available at http://www.wisa.co.za

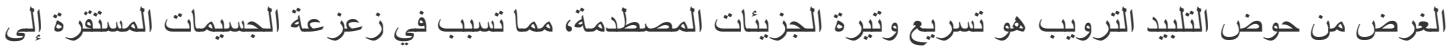

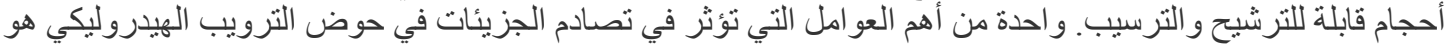

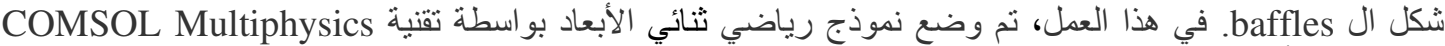

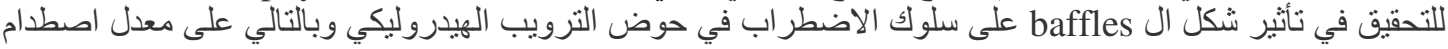

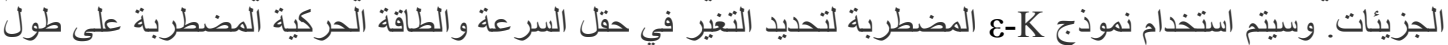

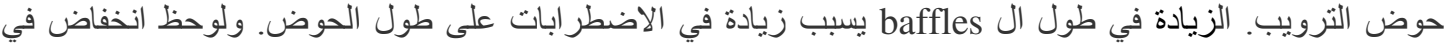

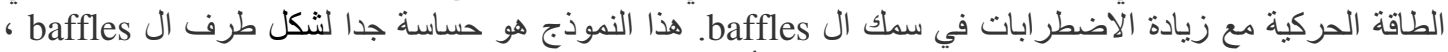

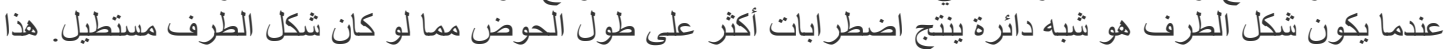

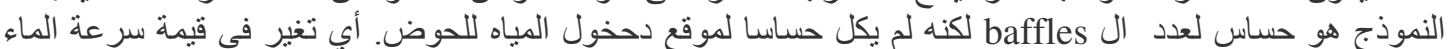

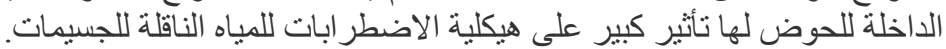

